\title{
Learning Graph Model for Different Dimensions Image Matching
}

\author{
Haoyi Zhou ${ }^{1}$, Xiao Bai ${ }^{1}$ and Jun Zhou ${ }^{2}$ \\ ${ }^{1}$ School of Computer Science and Engineering, \\ Beihang University XueYuan Road No.37, HaiDian District, Beijing, China \\ \{Haoyi, baixiao\}@buaa.edu.cn \\ ${ }^{2}$ School of Information and Communication Technology, \\ Griffith University, Nathan, QLD 4111, Australia \\ jun.zhou@griffith.edu.au
}

\begin{abstract}
Hyperspectral imagery has been widely used in real applications such as remote sensing, agriculture, surveillance, and geological analysis. Matching hyperspectral images is a challenge task due to the high dimensional nature of the data. The matching task becomes more difficult when images with different dimensions, such as a hyperspectral image and an RGB image, have to be matched. In this paper, we address this problem by investigating structured support vector machine to learn graph model for each type of image. The graph model incorporates both low-level features and stable correspondences within images. The inherent characteristics are depicted by using graph matching algorithm on weighted graph models. We validate the effectiveness of our method through experiments on matching hyperspectral images to RGB images, and hyperspectral images with different dimensions.
\end{abstract}

Keywords: Matching, Graph Model, Hyperspectral Image

\section{Introduction}

With the development of imaging technology, images can be acquired in different modalities, such as RGB image, radar image, IR image, UV image, hyperspectral image, and so on. Robust matching algorithms are needed for the purpose of fusion and registration between these images which have disparity in numbers of dimensions. The difficulties come from distinctive representation of image content in multi-modal images.

In this paper, we take hyperspectral image as an example of multi-dimensional data and investigate matching methods in two settings, i.e., matching hyperspectral image and RGB image, and matching hyperspectral images with different dimensions. 


\subsection{Motivation}

A hyperspectral image consists of grayscale bands each of which covers a small range of the light wavelengths ${ }^{1}$. We study the hyperspectral image matching problem from three aspects:

(1) Correspondence Our method is proposed based on the observation that local invariant features are interrelated among different hyperspectral/band images. That is to say, steady points and spatial features or structures [19] within or across bands of a hyperspectral image shall also be observable in other hyperspectral images, their spectral bands, or RGB images of the same scene.

(2) Graph Model Image matching is defined as finding the correct relationships between two point sets. To this end, many methods based on image keypoints have been proposed $[15,16]$. Because the internal structure of objects turns out to be important, the matching problem can be formulated as a graph matching problem by building and matching graphs from keypoints in each image [6]. Current graph methods cannot solve the matching problem effectively when images are in different dimensions. In this paper, we develop a uniform graph model that preserves stable correspondences between two images.

(3) Learning Method With a complete attributed graph [18] defined as the uniform model, the distinctive characteristic of each band affects the attribute of every vertex and influences the connections represented by edges. It is reasonable to adopt a learning method on weighted attributed graph, aiming to select the most discriminative components and to boost the performance of matching function.
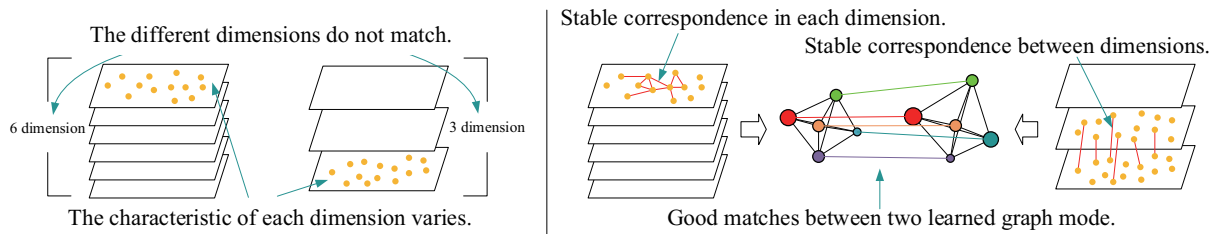

Fig. 1. Left Panel: difficulty in matching different dimensions. Right panel: our motivation (Change the description in the left panel to "6 dimensions", "3 dimensions", "Images with different dimensions do not match." Change the description in the right panel: "Good matches between two learned graph models.").

\subsection{Related Work}

We firstly review some recent work on matching or registration of multi-dimensional hyperspectral images. One active area that adopt this technique is painting conservation, which only permits "seeing" without "touching". New close range

\footnotetext{
${ }^{1}$ Hyperspectral images can be divided into two types, i.e., remote sensing and close range, according to the distance from the imaging device to the objects. We focus on the latter in this paper.
} 
hyperspectral sensors have been developed for this purpose [1,9]. While some approaches do not require image matching or calibration [11], methods based on local weighted mean measurements have achieved better performance in image registration [7]. However, these methods can only deal with image matching problem in constrained conditions.

Keypoint description methods, such SIFT[15] and SURF[2], have been used to match multi-dimensional images. However, a series of experiments show that they do not bring much advantages when matching across bands in wide wavelength range [19]. To get better results, Mukherjee et al proposed a method which merges the first few principal components of hyperspectral image in a Gaussian scale-space representation [17]. Meanwhile, a nonlinear combing function is used to get a scalar image along the spectral dimension using a scalar SIFT operator. Image matching performance can be further improved by developing better the keypoint detection approaches. For example, vector SIFT [8] operator extends SIFT based upon the multiple Gaussian scale space. Modified SIFT [20] descriptor preserves local edges with local contrast and differential excitation function. However, directly extending traditional image matching method into the spectral domain can not solve the complex hyperspectral image matching problem. In particular, the spatial correspondence among feature points can not be fully described in point-to-point matching prototype. Therefore, it is worthwhile to bring graph model into this problem so as to characterize the relationship between keypoints.

Our method requires the building of a complete graph and imposing edgepreserving constraints. Some basic and powerful matching algorithms have been proposed to serve for this purpose, such as Spectral Matching [13], Integer Projected Fixed Point Method [14], and Reweighted Random Walks [5]. Recently, Cho et al [3] parameterized the objective function of graph matching. This approach focuses on improving the matching results on similarity function of vertices. Our graph model is a weighted model defined on both edges and vertices. We followed the idea in [4] which formulates the graph matching problem into a structured output setting [21].

The main contribution of this paper are threefold. First, we present a novel method for matching images with different dimensions, which is far from being well investigated. Second, a novel graph learning model is proposed to extract stable correspondences among multi-dimensional images. Third, a formulation of structured output learning is redefined to deal with various correspondences.

\section{Methodology}

This section contains formulas and definition of three key steps of our method, i.e., graph construction, parameterization, and learning. 


\subsection{Graph Construction}

In this step, we construct a complete graph. Two important issues need to be addressed here, sort out vital feature points as vertices, and build finite but effective correspondences.

(1) Limited Feature Points We use the SIFT [15] or ("and" or "or"?) Hessian[16] detectors to detect local feature points in each band image. Known from [19], only repeatedly detected feature points are robust for cross band matching. Moreover, the graph will be too complex to match if too many keypoints are selected. Therefore, a k-means clustering method is performed on all points (based on their location or intensity?), and only $k$ keypoints that are closest to the clustering centers in each dimension are selected. An undirected graph $G_{i}$, where $i$ indexes the band number, is constructed from the selected keypoints in each dimension. As a consequence, a $D$-band hyperspectral image generates a graph set $\mathbf{G}=\left\{G_{1}, G_{2}, \ldots, G_{D}\right\}$.

(2) Various Correspondence The relevance between different bands can be captured by directly matching the spatial positions of the keypoints based on their feature descriptor using robust matching approach such as RANSAC [10]. We store the pair-wise points matching results between adjacent bands in $C_{i}$. Thus, various correspondences $C_{1}, C_{2}, \ldots, C_{M}$ form a set $\mathbf{C}$ (M=D-1 as only adjacent bands are matched?).

(3) Landmark Points Manually labelled landmark points can be treated as another type of correspondence. A manually selected subset of feature points are collected on each band of hyperspectral images. Then, we carefully mark the pair-wise matching keypoints between adjacent bands, generating a set $\mathbf{L}$, which is similar to $\mathbf{C}$ (i.e., $\mathbf{L}$ is a subset of $\mathbf{C}$ ?).

\subsection{Graph Matching Formulation}

To learn a graph model on exact undirected graph matching, we firstly review the graph formulation. An attributed graph [18] is represented as $G=(V, E, A)$ where $V$ contains nodes $v_{i}$ in the graph, $E$ consists of edges $e_{i j}$ between vertices. An attribute $a_{i i}$ is assigned to each node and $a_{i j}$ to each edge, which are real numbers or vectors, respectively.

Considering the matching problem between two attributed graphs $G=(V, E, A)$ and $G^{\prime}=\left(V^{\prime}, E^{\prime}, A^{\prime}\right)$, an assignment matrix $\mathbf{X} \in\{0,1\}^{N \times N^{\prime}}$ defines one of the possible matching correspondences, where $N=|V|$ and $N^{\prime}=\left|V^{\prime}\right|$ are the number of vertices in each graph. $x_{i j} \in \mathbf{X}$ should satisfy the following condition

$$
x_{i j}=\left\{\begin{array}{l}
1, \text { if } v_{i} \in V \text { matches } v_{j} \in V^{\prime} \\
0, \text { otherwise }
\end{array}\right.
$$

In this paper, we follow $[5,6,3,4]$ and denote $x \in\{0,1\}^{N N^{\prime}}$ as a column-wise vectorized replica of $\mathbf{X}$. A score function $S\left(G, G^{\prime}, x\right)$ represents the similarity of graph attributes. So the graph matching problem can be formulated using this 
maximizing score function $S\left(G, G^{\prime}, x\right)$ for possible assignment vector $x$ :

$$
\begin{aligned}
& x^{*}=\underset{x}{\operatorname{argmax}} S\left(G, G^{\prime}, x\right) \\
& \text { s.t. } x \in\{0,1\}^{N N^{\prime}}, \sum_{i=1}^{N} x_{i j} \leq 1, \sum_{j^{\prime}=1}^{N^{\prime}} x_{i^{\prime} j^{\prime}} \leq 1
\end{aligned}
$$

where constraints in Eq. (2) is required by the one-to-one matching between $G$ and $G^{\prime}$. Then the score function $S\left(G, G^{\prime}, x\right)$ is re-written to transform Eq. (1) into a manageable form. A similarity matrix $\mathbf{M}$ of $N N^{\prime} \times N N^{\prime}$ is introduced where the non-diagonal element $\mathbf{M}_{i j ; p q}=s_{E}\left(a_{i p}, a_{j q}^{\prime}\right)$ preserves the edge similarity between edge $e_{i p}$ in $G$ and $e_{j q}$ in $G^{\prime}$. The diagonal term $\mathbf{M}_{i j ; i j}=s_{V}\left(a_{i}, a_{j}^{\prime}\right)$ contains the node similarity between vertices $v_{i}$ in $G$ and $v_{j}$ in $G^{\prime}$. As a result, the score function is updated as:

$$
\begin{aligned}
S\left(G, G^{\prime}, x\right) & =\sum_{x_{i j}=1} s_{V}\left(a_{i}, a_{j}^{\prime}\right)+\sum_{x_{i j}=1, x_{p q}=1} s_{E}\left(a_{i p}, a_{j q}\right) \\
& =x^{T} \mathbf{M} x
\end{aligned}
$$

The graph matching problem in Eq. (1) is formulated as Integer Quadratic Program (IQP) problem known to be NP hard in Eq. (3).

\subsection{Parameterized Graph Model}

Given a multi-band image, an attributed graph $G_{i}$ is constructed on each band using the above steps. We can treat each $G_{i}$ as a weaker sample of the potentially optimized graph model. The correspondences $C_{i} \in \mathbf{C}$ with respect to $G_{i}$ are crucial clues in finding such a model.

To address the problem of learning graphs, we assumed that there exist a graph model $G^{*}$ that fits various correspondences. The matching between $G^{*}$ and graph $G_{i}$ of each band can be recovered by generating an assignment vector $x$ which reflects the correspondence maps $G^{*}$ to $\mathbf{G}$. Therefore, inspired by the structured output framework [21] and graph learning [3,12], we use the score function $S\left(G, G^{*}, x\right)$ as a compatibility function, getting scores of all possible assignment vectors from correspondence set $\mathbf{C}$. The problem of finding the best graph match from Eq.(2) can be expressed as:

$$
x^{*}\left(G^{*}, \beta ; G\right)=\underset{x \in \S_{1}\left(C_{G}\right)}{\operatorname{argmax}} S\left(G^{*}, G, x ; \beta\right)
$$

where $\beta$ is a weighting vector defined in [4], which indicates the importance of vertices and edges. $\S_{1}(\cdot)$ denotes the assignment vector generated from the first matching results in correspondence set $\mathbf{C}$. The learning process is performed on the labeled landmark? examples $\mathbf{Y}=\left\{\left(G_{1}, x_{1}\right), \ldots,\left(G_{d}, x_{d}\right)\right\}$ (Do you mean $\mathbf{L}$ defined in section 2.1), where $x_{i}=\S_{1}\left(C_{G_{i}}\right)$.

We parameterized both $G^{*}$ and $\beta$ in a vectorized form to gain a linear form of $S$. Following [4], the similarity functions $s_{V}$ and $s_{E}$ are dot products of two attribute vectors $s_{V}\left(a_{i}, a_{j}^{\prime}\right)=a_{i} \bullet a_{j}$ and $s_{E}\left(a_{i p}, a_{j q}^{\prime}\right)=a_{i p} \bullet a_{j q}$. Moreover, the 
attribute vector $\Theta\left(G^{*}\right)=\left[\cdots ; a_{i}^{*} ; \cdots ; a_{i j}^{*} ; \cdots\right]$ (Why do you have $a_{i}^{*}$ and $a_{i j}^{*}$, two different types of indices in a matrix (one subscript vs two subscripts)?). Feature map $\Psi(G, x)=\left[\cdots ; a_{\pi(i)}^{*} ; \cdots ; a_{\pi(i) \pi(j)}^{*} ; \cdots\right]$ are also defined in vectorized form (same question here), where $\pi(i)=j$ denotes a matching of $v_{i}^{*}$ in $G^{*}$ to $v_{j}$ in $G$ according to assignment vector $x$. With the requirement of max-margin framework [21], the scoring function in Eq. (4) can be factorized in a dot product form[4] $S\left(G^{*}, G, x ; \beta\right)=\mathbf{w} \bullet \Psi(G, x)$ (I removed $\langle$,$\rangle as \bullet$ is used as the symbol for dot product), where $\mathbf{w} \equiv \beta \odot \Theta\left(G^{*}\right)$ combines the weights $\beta$ and attributes $\Theta\left(G^{*}\right)(\odot$ denotes the Hadamard product). Then Eq. (4) can be rewritten in a linear form as follows

$$
x^{*}\left(G^{*}, \beta ; G\right)=\underset{x \in \S_{1}(G)}{\operatorname{argmax}} \mathbf{w} \cdot \Psi(G, x)
$$

Attributes Definition We represent edge attributes by a histogram of logpolar bins [4] to match the dot product assumption. The polar-angle of each edge is measured from the mean characteristic angle of all vertices in case there is no such angle. This will enhance the robustness of the method against rotation. Then, the SIFT descriptor [15] is adopted as the attribute of vertices for describing the local appearance.

\subsection{Graph Model learning}

Now, with the labeled examples $\mathbf{Y}=\left\{\left(G_{1}, x_{1}\right), \ldots,\left(G_{d}, x_{d}\right)\right\}$ (Again L?), a structured output maximum margin framework [21] can handle the learning of w. It can be applied to Eq. (5) and equivalently expressed in a margin re-scaling maximization formulation

$$
\begin{array}{ll}
\min _{\mathbf{w}, \xi} & \frac{1}{2}\|\mathbf{w}\|^{2}+\frac{\lambda}{d} \sum_{i=1}^{d} \xi_{i} \\
\text { s.t. }\left\{\begin{array}{l}
\forall i: \xi_{i} \geq 0 \\
\forall i, \forall x \in \S_{1}\left(C_{G}\right) \backslash x_{i}:\langle\mathbf{w}, \delta \Psi(G, x)\rangle \geq \Delta\left(x_{i}, x\right)-\xi_{i}
\end{array}\right.
\end{array}
$$

where $\delta \Psi(G, x) \equiv \Psi\left(G, x_{i}\right)-\Psi(G, x), \Delta\left(x_{i}, x\right)$ stands for penalty of loss function on choosing $x$ instead of the reliable assignment vector $x_{i}, \lambda$ is a parameter controlling the trade-off between regularization and loss terms.

Noticed that only the first correspondence $C_{1}$ in $\mathbf{C}$ is brought into the Eq. (5). However, there is always more than one correspondence besides the landmarks. Despite the fact that manually landmark labelling is time-consuming, we show that combining landmarks with various correspondences is an efficient way of improving the performance of the graph model. Thus, we add an additional set of constraints and modify Eq. (6) into

$$
\begin{array}{ll}
\min _{\mathbf{w}, \xi, \nu} & \frac{1}{2}\|\mathbf{w}\|^{2}+\frac{\lambda_{1}}{d} \sum_{i=1}^{d}\left(\xi_{i}+\lambda_{2} \nu_{i}\right) \\
\text { s.t. }\left\{\begin{array}{l}
\forall i: \xi_{i}, \nu_{i} \geq 0 \\
\forall i, \forall x \in \S_{1}\left(C_{G}\right) \backslash x_{i}:\langle\mathbf{w}, \delta \Psi(G, x)\rangle \geq \Delta\left(x_{i}, x\right)-\xi_{i} \\
\forall i, \forall x^{\prime} \in \S_{2}\left(C_{G}\right) \backslash x_{i}:\left\langle\mathbf{w}, \delta \Psi\left(G, x^{\prime}\right)\right\rangle \geq \Delta\left(x_{i}, x^{\prime}\right)-\nu_{i}
\end{array}\right.
\end{array}
$$


where $\lambda_{1}$ is the trade-off parameter, $\lambda_{2}$ controls the effect of second correspondence. More correspondences or manually labelled landmark can also be settled (produced?) in this way. The structured SVM [21] allows us to maximize the margin of the constraints in Eq. (7). The graph model contained in $\mathbf{w}$ is learned along with structured output. The initial graph model is set at median band as it matches other bands quite well in the experiments [19].

Loss Function A loss function is required in the objective function of Eq. (7) to measure the sensitivity of a predicted assignment vector $x^{*}$ against the pre-provide (initial?) correspondences in assignment vector $x$. We define the normalized Hamming loss as $\Delta\left(x_{i}, x\right)=1-\frac{x_{i} \cdot x}{\left\|x_{i}\right\|_{F}^{2}}$, where $\|\cdot\|_{F}$ denotes the Frobenius norm, on mismatch rate. This has been proved to be effective in graph matching method $[3,4]$.

\subsection{Matching Method}

Given an attribute graph $\mathrm{G}_{1}$ with weights $\mathrm{B}_{1}$ (pay attention to the font here, previously they are italic) is being generated on a multi-band image, we can perform the same steps on the target (different) band image to obtain a attribute graph $\mathrm{G}_{2}$ (again, font) and weights $\mathrm{B}_{2}$. The graph matching problem formulated in Eq. (2) and Eq. (3) can be parameterized with weights as:

$$
x^{*}=\underset{x}{\operatorname{argmax}} x^{T} \mathbf{M}_{\mathbf{w}} x \text { s.t. } x \in\{0,1\}^{N N^{\prime}}, \sum_{i=1}^{N} x_{i j} \leq 1, \sum_{j^{\prime}=1}^{N^{\prime}} x_{i^{\prime} j^{\prime}} \leq 1
$$

where $\mathbf{M}_{\mathbf{w}}=\left(\beta_{1} \odot \beta_{2}\right) \cdot \mathbf{M}$. So the traditional matching methods mentioned in Section 1.2 are elegant options to address this standard graph matching problem.

\section{Experiments}

We have applied our algorithm on multi-dimensional images in the Multispectral Image Database [22]. Fig. 2 shows two examples ${ }^{2}$.

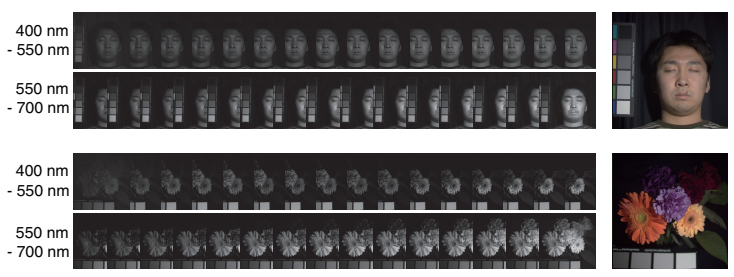

Fig. 2. Hyperspectral images (left) in the range from $400 \mathrm{~nm}$ to $700 \mathrm{~nm}$ with a step size of $10 \mathrm{~nm}$ and the corresponding RGB image (right).

\footnotetext{
${ }^{2}$ The dataset contains 32 scenes. Every scene contains a 31 band hyperspectral image and a composite RGB image. We selected 14 of them in various classes, including face, paints, toys, flowers and etc.
} 
Firstly, we generated two image sets from the 14 selected hyperspectral images. In set 1 , the first 25 bands of each image form a 25-dimensional image and the matching is performed on the rest 6 bands of the same scene. Then, we matched each 31-dimensional image with the corresponding RGB image in set 2. A tolerance $t=10$, approximately $2 \%$ of the band image size (512 pixels), is defined to determine the correctness of point-to-point matching. (If the number of mismatches is large than the tolerance, the matching is considered as incorrect.) The matching accuracy is calculated by $\frac{\sharp\{\text { correct matches }\}}{\sharp\{\text { all matches }\}}$, where $\sharp\{\cdot\}$ denotes the number of elements (what do you mean elements? keypoints or all pixels?). Landmarks were manually marked on 10 selected points for adjacent bands.

The SIFT [15] and vector SIFT[8] matching algorithms were applied between every band (or neighboring bands?) of images pairs. We calculated the mean accuracy and record the worst case.

Table 1. Matching accuracy on set 1 (25 band images to 6 band images).

\begin{tabular}{l|cccccccc|c}
\hline \hline Methods & face & face $^{\dagger}$ & watercolors watercolors & $\dagger$ & flowers & flowers & toys toys & Average \\
\hline SIFT(average) & 73.8 & 72.1 & 80.9 & 80.5 & 89.4 & 89.3 & 87.5 & 85.6 & 73.7 \\
SIFT(worst) & 35.7 & 30.8 & 50.1 & 44.4 & 79.9 & 78.2 & 78.0 & 73.0 & - \\
Vector SIFT & 81.3 & 74.2 & 82.3 & 78.9 & 93.1 & $\mathbf{9 0 . 4}$ & 85.1 & 81.4 & 78.1 \\
our+SM & 83.3 & 80.3 & $\mathbf{9 6 . 7}$ & $\mathbf{9 3 . 3}$ & 86.7 & 86.7 & 90.0 & 87.7 & 87.9 \\
our+RRWM & $\mathbf{8 6 . 7}$ & $\mathbf{8 1 . 1}$ & $\mathbf{9 6 . 7}$ & 90.0 & $\mathbf{9 3 . 3}$ & 90.2 & $\mathbf{9 3 . 3}$ & $\mathbf{9 2 . 8}$ & $\mathbf{8 8 . 8}$ \\
\hline \hline
\end{tabular}

The experimental results on set 1 are shown in Table 1 . The accuracy of keypoint based method various significantly. The worst case is observed in matching bands that are apart to each other, as reported in [19]. Setting $k=30$ and $\lambda=0.25$, we obtain novel graph models for individuals (what do you mean individuals?). Using matching method in [13] and [5], our proposed methods outperform the traditional matching methods on the average results and avoid the worst case. Accuracy varies in less extent on our matching methods. In addition, the face $e^{\dagger}$ represents 12 rotated scenes from $0^{\circ}$ to $360^{\circ}$. Resulting from using the same descriptors, our method and SIFT approach show similar pattern in decrease of accuracy (You did not show results on this decrease pattern, better remove the statement.).

Table 2. Matching accuracy on set 2 (31 band images to RGB images)

\begin{tabular}{l|cccccccc|c}
\hline \hline Methods & \multicolumn{1}{|c}{ face } & face $^{\dagger}$ & watercolors watercolors & & flowers & flowers & toys & toys & Average \\
\hline SIFT(average) & 83.1 & 78.1 & 82.1 & 82.2 & 91.5 & 91.3 & 75.9 & 74.1 & 79.6 \\
SIFT(worst) & 55.5 & 41.2 & 73.8 & 73.2 & 81.5 & 80.8 & 69.1 & 34.3 & - \\
Vector SIFT & 78.2 & 76.6 & 84.2 & 83.2 & 92.7 & 90.4 & 76.2 & 74.1 & 81.9 \\
our+SM & 80.0 & 73.3 & 90.0 & 86.9 & 90.0 & 89.1 & 86.7 & 83.3 & 85.0 \\
our+RRWM & 80.0 & 76.9 & $\mathbf{9 3 . 3}$ & 86.7 & $\mathbf{9 6 . 7}$ & 90.0 & 88.1 & 86.7 & 85.7 \\
our $^{R}+$ RRWM & 83.3 & 79.7 & 86.7 & 86.7 & 93.3 & $\mathbf{9 2 . 2}$ & 90.0 & 90.0 & 89.5 \\
our $^{R L}+$ RRWM & $\mathbf{9 3 . 3}$ & $\mathbf{9 0 . 2}$ & 90.0 & $\mathbf{8 9 . 7}$ & 90.0 & 86.7 & $\mathbf{9 3 . 3}$ & $\mathbf{9 1 . 4}$ & $\mathbf{9 1 . 3}$ \\
\hline \hline
\end{tabular}


The experimental results on set 2 are presented in Table 2. Compared with previous experiment, the accuracies decrease slightly due to the bigger gap between RGB images and hyperspectral images. But our method still demonstrate improvement over the alternative methods.

Moreover, we applied random rotations $\mathbb{R}_{i}$ in the range of $\left[0^{\circ}, 360^{\circ}\right]$ on labeled examples $\mathbf{Y}$ (landmarks L?) to demonstrate the anti-rotation ability of graph models. The results are shown on the penultimate (last two rows?) row in Table 2. The rotation generates some negative influences on matching outcomes, e.g., in watercolors and flowers.

The method our ${ }^{R L}$ introduces landmark correspondences $\mathbf{L}$ as the second correspondence into the structured output max-margin framework in Eq. (7) with $\lambda_{2}=0.15$ and $\lambda_{3}=0.35$. The landmarks in face turn to be highly effective rather than in flowers and watercolors. The latter contains insignificant correspondences and complicated pieces, like petals, which are hard to recognize correctly.

Some visualized band matching results are shown in Fig. 3.
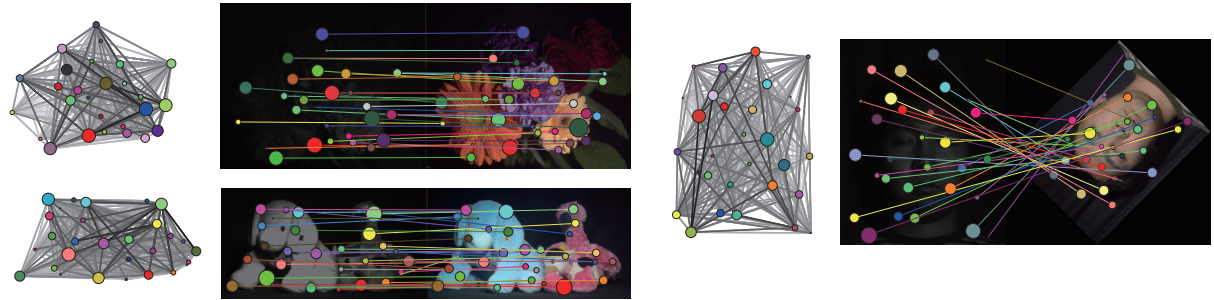

Fig. 3. Weighted Graph model for hyperspectral image(left) and matching between different band images (right).

\section{Conclusions}

Matching of images with different dimensions is a non-trivial problem. In this paper, we have propose a learning weighted graph model to catch the most discriminative feature points and correspondences among each band image. A structured output framework is applied to address the $N P$-Hard graph matching problem with multiple constraints (the phrase "with multiple constraints" is used to describe the structured output framework or graph matching problem?). The experiments show significant improvement on the matching performance between different bands, especially with few landmarks, when compared against several alternative methods.

\section{Acknowledgement}

This research is supported by the National Natural Science Foundation of China (NSFC) projects No.61370123 and No.61402389. 


\section{References}

1. Balas, C., Papadakis, V., Papadakis, N., Papadakis, A., Vazgiouraki, E., Themelis, G.: A novel hyper-spectral imaging apparatus for the non-destructive analysis of objects of artistic and historic value. Journal of Cultural Heritage 4, 330-337 (2003)

2. Bay, H., Ess, A., Tuytelaars, T., Van Gool, L.: Speeded-up robust features (SURF). Computer Vision and Image Understanding 110(3), 346-359 (2008)

3. Caetano, T.S., McAuley, J.J., Cheng, L., Le, Q.V., Smola, A.J.: Learning graph matching. IEEE Transactions on Pattern Analysis and Machine Intelligence 31(6), 1048-1058 (2009)

4. Cho, M., Alahari, K., Ponce, J.: Learning graphs to match. In: IEEE International Conference on Computer Vision. pp. 25-32. IEEE (2013)

5. Cho, M., Lee, J., Lee, K.M.: Reweighted random walks for graph matching. In: Computer Vision - ECCV 2010, vol. 6315, pp. 492-505. Springer (2010)

6. Cour, T., Srinivasan, P., Shi, J.: Balanced graph matching. Advances in Neural Information Processing Systems 19, 313 (2007)

7. Diem, M., Lettner, M., Sablatnig, R.: Multi-spectral image acquisition and registration of ancient manuscripts 224, 129-136 (2007)

8. Dorado-Muñoz, L.P., Velez-Reyes, M., Mukherjee, A., Roysam, B.: A vector SIFT operator for interest point detection in hyperspectral imagery. In: 2nd Workshop on Hyperspectral Image and Signal Processing: Evolution in Remote Sensing. pp. 1-4. IEEE (2010)

9. Easton Jr, R.L., Knox, K.T., Christens-Barry, W.A.: Multispectral imaging of the Archimedes palimpsest. In: IEEE Applied Imagery Pattern Recognition Workshop. pp. 111-111. IEEE Computer Society (2003)

10. Fischler, M.A., Bolles, R.C.: Random sample consensus: a paradigm for model fitting with applications to image analysis and automated cartography. Communications of the ACM 24(6), 381-395 (1981)

11. Fontana, R., Gambino, M.C., Greco, M., Marras, L., Pampaloni, E.M., Pelagotti, A., Pezzati, L., Poggi, P.: 2D imaging and 3D sensing data acquisition and mutual registration for painting conservation. In: Electronic Imaging 2005. pp. 51-58. International Society for Optics and Photonics (2005)

12. Hare, S., Saffari, A., Torr, P.H.: Efficient online structured output learning for keypoint-based object tracking. In: IEEE Conference on Computer Vision and Pattern Recognition. pp. 1894-1901. IEEE (2012)

13. Leordeanu, M., Hebert, M.: A spectral technique for correspondence problems using pairwise constraints. In: IEEE International Conference on Computer Vision. vol. 2, pp. 1482-1489. IEEE (2005)

14. Leordeanu, M., Hebert, M., Sukthankar, R.: An integer projected fixed point method for graph matching and map inference. In: Advances in Neural Information Processing Systems. vol. 22, pp. 1114-1122 (2009)

15. Lowe, D.G.: Distinctive image features from scale-invariant keypoints. International journal of computer vision 60(2), 91-110 (2004)

16. Mikolajczyk, K., Schmid, C.: Scale \& affine invariant interest point detectors. International journal of computer vision 60(1), 63-86 (2004)

17. Mukherjee, A., Velez-Reyes, M., Roysam, B.: Interest points for hyperspectral image data. IEEE Transactions on Geoscience and Remote Sensing 47(3), 748-760 (2009)

18. Pelillo, M.: A unifying framework for relational structure matching. In: Fourteenth International Conference on Pattern Recognition. vol. 2, pp. 1316-1319. IEEE (1998) 
19. Saleem, S., Bais, A., Sablatnig, R.: A performance evaluation of SIFT and SURF for multispectral image matching. In: Image Analysis and Recognition, pp. 166173. Springer (2012)

20. Saleem, S., Sablatnig, R.: A modified SIFT descriptor for image matching under spectral variations. In: International Conference on Image Analysis and Processing, pp. 652-661. Springer (2013)

21. Tsochantaridis, I., Joachims, T., Hofmann, T., Altun, Y.: Large margin methods for structured and interdependent output variables. In: Journal of Machine Learning Research. pp. 1453-1484 (2005)

22. Yasuma, F., Mitsunaga, T., Iso, D., Nayar, S.K.: Generalized assorted pixel camera: postcapture control of resolution, dynamic range, and spectrum. IEEE Transactions on Image Processing 19(9), 2241-2253 (2010) 